\title{
Energy levels, radiative rates and excitation rates for transitions in $\mathrm{Ni} \mathrm{XI}^{\star}$
}

\author{
K. M. Aggarwal and F. P. Keenan
}

\author{
Astrophysics Research Centre, School of Mathematics and Physics, Queen's University Belfast, Belfast BT7 1NN, \\ Northern Ireland, UK \\ e-mail: K.Aggarwal@qub.ac.uk
}

Received 28 August 2007 / Accepted 17 September 2007

\begin{abstract}
Aims. In this paper we report calculations for energy levels, radiative rates and excitation rates for transitions in Ni XI. Methods. The GRASP (General-purpose Relativistic Atomic Structure Package) and FAC (Flexible Atomic Code) have been adopted for calculating energy levels and radiative rates, and the Dirac Atomic R-matrix Code (DARC) has been used to determine the excitation rates.

Results. Oscillator strengths, radiative rates and line strengths are reported for all E1, E2, M1 and M2 transitions among the lowest 250 levels of Ni XI. Additionally, lifetimes are also reported for all levels. However, results for excitation rates are presented only for transitions among the lowest 17 levels.
\end{abstract}

Key words. atomic data, atomic processes

\section{Introduction}

Iron group elements $(\mathrm{Sc}-\mathrm{Zn})$ are becoming increasingly important in astrophysical plasmas, as many of their emission lines are frequently observed from different ionisation stages. These observations provide a wealth of data about the plasma characteristics, such as: temperature, density and chemical composition. Additionally, iron group elements are often impurities in fusion reactors, and to estimate the power loss from the impurities, atomic data (namely energy levels, oscillator strengths or radiative decay rates, and excitation rates) are required for many ions. Since there is paucity of measured parameters, one must depend on theoretical results. Therefore, recently we have reported atomic parameters for many ions of Fe (IX-XXVI; see Aggarwal \& Keenan 2006, and references therein). Nickel is the second most abundant element (after iron) among the iron group in solar plasmas, and therefore we have also reported atomic data for many of its ionisation stages (XIII-XIX; see Aggarwal et al. 2007, and references therein). In this paper we focus our attention on Ar-like Ni XI.

Emission lines of Ni XI have been observed in the solar corona by Feldman et al. (1965), Wagner \& House (1971), Svensson et al. (1974), Magnant-Crifo (1975), Edlen \& Smitt (1978), and Sandlin \& Tousey (1979). Its lines have also been measured in laboratory plasmas by Fawcett et al. (1972), and identified in the soft X-ray region (5-175 $\AA$ ) in the F-type stars Procyon and $\alpha$ Centauri by Raassen et al. $(2002,2003)$ from spectra obtained by the Chandra and XMM-Newton satellites. Similarly, spectra in the 14.4-16.5 nm wavelength range in high temperature JET tokamak plasmas have revealed many emission lines from Ni ions, including Ni XI (Mattioli et al. 2004).

$\star$ Tables 1, 3, 4 and 7 are only available in electronic form at the CDS via anonymous ftp to cdsarc.u-strasbg.fr (130.79.128.5) or via

http://cdsweb.u-strasbg.fr/cgi-bin/qcat?J/A+A/475/393
In spite of the diagnostic potential of $\mathrm{Ni} \mathrm{XI}$ lines and the requirement for modelling applications, there are only a few calculations of atomic data available in the literature. Most of the calculations are confined to the determination of energy levels and radiative rates ( $A$-values), such as by Wagner \& House (1971). A more recent but unpublished calculation is by Irimia \& Froese-Fischer (2003), who adopted the multi-configuration Hartree-Fock (MCHF) code, and their results are available at the website http://www.vuse.vanderbilt.edu/ cff/ mchf_collection/. Furthermore, very recently Verma et al. (2007) have reported results for energy levels, radiative rates, collision strengths $(\Omega)$, and effective collision strengths $(\Upsilon)$ for transitions among the lowest 17 levels of the $\left(1 s^{2} 2 s^{2} 2 p^{6}\right)$ $3 s^{2} 3 p^{6}, 3 s^{2} 3 p^{5} 3 d$ and $3 s^{2} 3 p^{6} 3 d$ configurations of Ni XI. They have adopted the cIV3 code of Hibbert (1975) for the generation of wavefunctions, and the R-matrix code of Berrington et al. (1995) for the calculations of $\Omega$ and $\Upsilon$. Additionally, they have included configuration interaction $(\mathrm{CI})$ as well as one-body relativistic operators for calculating the energy levels and $A$-values. Similarly, they have resolved resonances in the thresholds region in order to determine the values of $\Upsilon$ over a wide temperature range up to $10^{7} \mathrm{~K}$. Unfortunately, their results have been found to be unreliable for all parameters, as discussed and demonstrated by Aggarwal \& Keenan (2007). Therefore, we will not discuss their atomic data any further in this paper.

In this paper we have adopted the GRASP (General-purpose Relativistic Atomic Structure Package), originally developed as GRASP0 by Grant et al. (1980) and a revised and modified version was published as GRASP1 by Dyall et al. (1989). This code has been further updated by Dr. P. H. Norrington. It is a fully relativistic code, and is based on the $j j$ coupling scheme. Further relativistic corrections arising from the Breit interaction and QED effects have also been included. Additionally, we have used the option of extended average level (EAL), in which a weighted (proportional to $2 j+1$ ) trace of the Hamiltonian 
matrix is minimized. This produces a compromise set of orbitals describing closely lying states with moderate accuracy. Furthermore, in order to assess the accuracy of our results we have performed parallel calculations from the Flexible Atomic Code (FAC) of $\mathrm{Gu}$ (2003), which is available from the website http://kipac-tree.stanford.edu/fac. This is also a fully relativistic code which provides a variety of atomic parameters, and yields results comparable to GRASP, as already shown for three Mg-like ions by Aggarwal et al. (2007). Thus results from FAC will be helpful in assessing the accuracy of our energy levels and radiative rates.

\section{Energy levels}

The $3 s^{2} 3 p^{6}, 3 s^{2} 3 p^{5} 3 d$ and $3 s 3 p^{6} 3 d$ configurations of Ni XI give rise to the lowest 17 levels discussed in our earlier paper (Aggarwal \& Keenan 2007). Just above the highest level $\left({ }^{1} D_{2}\right)$ of the $3 \mathrm{~s} 3 \mathrm{p}^{6} 3 \mathrm{~d}$ configuration (see Table 1) lie 111 levels of the $3 \mathrm{p}^{4} 3 \mathrm{~d}^{2}$ configuration, and some of these intermix with the 90 levels of $3 \mathrm{~s}^{3} \mathrm{p}^{5} 3 \mathrm{~d}^{2}$, which further interact with the 350 levels of $3 s^{2} 3 p^{3} 3 d^{3}$ and 522 levels of $3 s 3 p^{4} 3 d^{3}$. Therefore, a calculation with 1090 levels of the $3 s^{2} 3 p^{6}, 3 s^{2} 3 p^{5} 3 d, 3 s 3 p^{6} 3 d, 3 p^{4} 3 d^{2}$, $3 \mathrm{~s} 3 \mathrm{p}^{5} 3 \mathrm{~d}^{2}, 3 \mathrm{~s}^{2} 3 \mathrm{p}^{3} 3 \mathrm{~d}^{3}$, and $3 \mathrm{~s}^{3} \mathrm{p}^{4} 3 \mathrm{~d}^{3}$ configurations becomes (almost) essential in order to improve the accuracy of energy levels and $A$-values, as already demonstrated in our earlier paper. Apart from achieving a better accuracy, a larger calculation is required for modelling purpose as emphasized and demonstrated by Liedahl (2000). Furthermore, transitions involving the higher excited levels of the $n=4$ configurations have already been observed in solar plasmas (Wagner \& House 1971) as well as laboratory sources (Fawcett et al. 1972), and therefore there is a clear need to include the $n=4$ configurations in generating the atomic data. Subsequently, we have included the additional four configurations $\left(3 s^{2} 3 p^{5} 4 \ell\right)$, which give rise to only 38 levels, but closely interact with the levels of the above listed seven $n=3$ configurations. This calculation includes a total of 1128 levels, but results are only presented for the lowest 250 levels for the sake of brevity. These results include all levels of the $3 \mathrm{~s}^{2} 3 \mathrm{p}^{6}$, $3 s^{2} 3 p^{5} 3 d, 3 s 3 p^{6} 3 d, 3 p^{4} 3 d^{2}, 3 s^{2} 3 p^{5} 4 s$ and $3 s^{2} 3 p^{5} 4 p$ configurations, and a complete set of atomic data for all levels may be obtained on request from KMA (K.Aggarwal@qub.ac.uk).

In Table 1 we compare our level energies from GRASP, obtained with and without the inclusion of Breit and QED effects. The inclusion of these effects lowers the level energies by a maximum of 0.03 Ryd (or equivalently $\leq 0.25 \%$ ), see for example, levels $30\left(3 \mathrm{p}^{4}\left({ }^{3} \mathrm{P}\right) 3 \mathrm{~d}^{2}{ }^{5} \mathrm{G}_{5}\right), 112\left(3 \mathrm{p}^{4}\left({ }^{1} \mathrm{~S}\right) 3 \mathrm{~d}^{2}{ }^{1} \mathrm{G}_{4}\right)$, and $179\left(3 \mathrm{p}^{3}\left({ }^{4} \mathrm{~S}\right) 3 \mathrm{~d}^{3}{ }^{7} \mathrm{~F}_{6}^{\circ}\right)$. Apart from this, the level orderings are slightly altered in a few instances, such as for levels $78 / 79$, $159 / 160$, and $212 / 213$. However, the energy difference between any two swapping levels is $\leq 0.01$ Ryd. Similarly, our other calculation performed with FAC and including the same CI as in GRASP, provides comparable energy levels within 0.1 Ryd, which is highly satisfactory. However, the level orderings are slightly different in a few instances, such as for levels $48 / 49$, $54 / 55$ and 78/79. All such levels are close to each other, and the FAC orderings are generally in better agreement with our corresponding results obtained without the inclusion of Breit and QED effects.

Experimental energies are available only for the levels of the $3 s^{2} 3 p^{6}, 3 s^{2} 3 p^{5} 3 d$, and $\left(3 s^{2} 3 p^{5}\right) 4 s$, $4 d$ and $4 f$ configurations. In our earlier paper (Aggarwal \& Keenan 2007), we have made extensive comparisons for the energy levels of the $3 s^{2} 3 p^{6}$ and $3 s^{2} 3 p^{5} 3 d$ configurations, and hence will not repeat these here. However, in Table 2 we compare our energies for the levels of the $3 s^{2} 3 p^{5} 4 \ell$ configurations with the experimentally compiled results of NIST (National Institute for Standards and Technology), available at their website http://physics.nist.gov/PhysRefData, and the unpublished results of Irimia \& Froese-Fischer (2003) for the levels of the $3 s^{2} 3 p^{5} 4 s$ configuration, which have been obtained from their multi-configuration Hartree-Fock (MCHF) code, and are available at the website listed above. Also included in this table are the earlier calculations of Wagner \& House (1971) for the levels of the $3 s^{2} 3 p^{5} 4 f$ configuration.

Our level energies from GRASP are higher than the experimental ones by $\sim 0.5 \mathrm{Ryd}$, but the ordering is the same. Similarly, the level energies from FAC are lower than from GRASP by $\sim 0.1$ Ryd, and hence are comparatively closer to the experimental values. However, the energy levels obtained with larger CI from the 6202 level FAC calculation (described later) differ by negligible amounts from those with lesser CI, i.e. with only 1128 levels. This indicates that the additional inclusion of CI is of no appreciable advantage. On the other hand, the MCHF calculations of Irimia \& Froese-Fischer (2003) for the levels of the $3 s^{2} 3 p^{5} 4 s$ configuration are not only (slightly) lower than those from GRASP and FAC, but the degeneracy among the levels is also minimal. Similarly, the differences between the calculations of Wagner \& House (1971) for the levels of the $3 s^{2} 3 p^{5} 4 f$ configuration and our results from GRASP and FAC are smaller than 0.6 Ryd, but their orderings are different from the experimental or our theoretical results, particularly for the ${ }^{3} \mathrm{~F}_{2}(555)$ and ${ }^{1} \mathrm{G}_{4}$ (569) levels. However, we would like to emphasize here that identifying a level in an atomic structure calculation is not always straightforward, as the eigenvector from the same level/configuration may dominate for two (or even more) levels. For this reason we have also included the mixing coefficients of the levels in Table 2, and examples of strong mixing are the levels $(4 s){ }^{3} \mathrm{P}_{1}^{\circ}$ and ${ }^{1} \mathrm{P}_{1}^{\circ}(133,143),(4 \mathrm{p}){ }^{3} \mathrm{D}_{2}$ and ${ }^{1} \mathrm{D}_{2}(186,212)$, and $(4 \mathrm{f}){ }^{3} \mathrm{~F}_{2}^{\circ}$ and ${ }^{1} \mathrm{D}_{2}^{\circ}(358,387)$. Therefore, the configuration and the $J$ values given in Table 1 (and 2) are definitive, but the corresponding $L S J$ designation provided for a level is only for guidance, and is liable to interchange. Additionally, for highly mixed levels the $L S J$ designations provided in Table 1 are not fully appropriate and the $j j$ coupling scheme, as adopted in the calculations, is more suitable. Therefore, in Table 1 we have also provided the corresponding $j j$ designations to facilitate the level/configuration identifications.

Assessing the accuracy of energy levels is not a simple task, especially when the corresponding data from experimental or other theoretical work are not already available for a majority of the levels. Nickel is a comparatively heavy ion and therefore relativistic effects are important, as included in the present work. Similarly, for many $\mathrm{Ni}$ ions the inclusion of extensive CI is also important, as already demonstrated for the lowest 17 levels of Ni XI (Aggarwal \& Keenan 2007), and for the levels of Ni XIII-XVII by Aggarwal et al. (2003, 2007). However, we noted above in Table 2 that additional CI over that included in our GRASP calculations is of no appreciable advantage as far as the levels of the $3 s^{2} 3 p^{5} 4 \ell$ configurations are concerned. Most of these are higher excited levels which are not included in Table 1. Therefore, to assess the accuracy of the lowest 250 levels, we have included in Table 1 our energies obtained from the larger calculation from FAC. This calculation includes 6202 levels from all possible $n=3$ plus $3 \mathrm{~s}^{2} 3 \mathrm{p}^{5} 4 \ell$ configurations. A comparison between the two sets of calculations from FAC shows a maximum discrepancy of $0.3 \mathrm{Ryd}(\leq 3 \%)$ for some of the levels, such as $100-120$, and the results obtained from the larger calculation are comparatively lower and hence closer to the corresponding 
Table 2. Energy levels (in Ryd) of the $3 \mathrm{~s}^{2} 3 \mathrm{p}^{5} 4 \ell$ configurations of Ni XI.

\begin{tabular}{|c|c|c|c|c|c|c|c|c|}
\hline$\overline{\text { Index }}$ & Configuration & Level & NIST & $\overline{\text { GRASP }}$ & FAC1 & FAC2 & MCHF & $\overline{\text { Mixing Coefficients }}^{a}$ \\
\hline 132 & $4 \mathrm{~s}$ & ${ }^{3} \mathrm{P}_{2}^{\circ}$ & & 11.9619 & 11.8806 & 11.8753 & 11.7411 & $0.997(132)$ \\
\hline 133 & $4 \mathrm{~s}$ & ${ }^{3} \mathrm{P}_{1}^{\circ}$ & 11.5725 & 12.0238 & 11.9414 & 11.9362 & 11.7444 & $0.772(133), 0.632(143)$ \\
\hline 140 & $4 \mathrm{~s}$ & ${ }^{3} \mathrm{P}_{0}^{\circ}$ & & 12.1785 & 12.0954 & 12.0898 & 11.7496 & $0.995(140)$ \\
\hline 143 & $4 \mathrm{~s}$ & ${ }^{1} \mathrm{P}_{1}^{\circ}$ & 11.7746 & 12.2332 & 12.1487 & 12.1433 & 11.7536 & $-0.774(143), 0.629(133)$ \\
\hline 180 & $4 p$ & ${ }^{3} \mathrm{~S}_{1}$ & & 12.9331 & 12.8574 & 12.8504 & & $-0.951(180)$ \\
\hline 186 & $4 \mathrm{p}$ & ${ }^{3} \mathrm{D}_{2}$ & & 13.0644 & 12.9849 & 12.9774 & & $0.804(186), 0.522(212)$ \\
\hline 187 & $4 p$ & ${ }^{3} \mathrm{D}_{3}$ & & 13.0858 & 13.0065 & 12.9995 & & $0.997(187)$ \\
\hline 195 & $4 \mathrm{p}$ & ${ }^{3} \mathrm{D}_{1}$ & & 13.1373 & 13.1011 & 13.0494 & & $-0.668(195), 0.583(208), 0.436(214)$ \\
\hline 202 & $4 \mathrm{p}$ & ${ }^{3} \mathrm{P}_{2}$ & & 13.1925 & 13.1416 & 13.1031 & & $0.796(202), 0.587(212)$ \\
\hline 208 & $4 \mathrm{p}$ & ${ }^{1} \mathrm{P}_{1}$ & & 13.2799 & 13.1979 & 13.1915 & & $0.719(195), 0.651(208)$ \\
\hline 210 & $4 \mathrm{p}$ & ${ }^{3} \mathrm{P}_{0}$ & & 13.3035 & 13.2197 & 13.1273 & & $0.974(210)$ \\
\hline 212 & $4 \mathrm{p}$ & ${ }^{1} \mathrm{D}_{2}$ & & 13.3590 & 13.2765 & 13.2688 & & $-0.611(212), 0.578(186), 0.534(202)$ \\
\hline 214 & $4 \mathrm{p}$ & ${ }^{3} \mathrm{P}_{1}$ & & 13.3696 & 13.2860 & 13.2785 & & $0.814(214),-0.476(208)$ \\
\hline 249 & $4 \mathrm{p}$ & ${ }^{1} \mathrm{~S}_{0}$ & & 13.8224 & 13.7346 & 13.6888 & & $0.919(249)$ \\
\hline 337 & $4 d$ & ${ }^{3} \mathrm{P}_{0}^{\circ}$ & & 14.6543 & 14.5779 & 14.5846 & & $-0.965(337)$ \\
\hline 342 & $4 d$ & ${ }^{3} \mathrm{P}_{1}^{\circ}$ & 14.3188 & 14.6704 & 14.5983 & 14.6022 & & $0.863(342)$ \\
\hline 348 & $4 d$ & ${ }^{3} \mathrm{P}_{2}^{\circ}$ & & 14.7069 & 14.6381 & 14.6430 & & $0.753(348)$ \\
\hline 350 & $4 d$ & ${ }^{3} \mathrm{~F}_{4}^{\circ}$ & & 14.7196 & 14.6341 & 14.6284 & & $0.970(350)$ \\
\hline 353 & $4 d$ & ${ }^{3} \mathrm{~F}_{3}^{\circ}$ & & 14.7288 & 14.6331 & 14.6485 & & $0.684(353), 0.529(392)$ \\
\hline 358 & $4 d$ & ${ }^{3} \mathrm{~F}_{2}^{\circ}$ & & 14.7810 & 14.7037 & 14.7054 & & $-0.655(358), 0.568(387), 0.436(393)$ \\
\hline 360 & $4 d$ & ${ }^{3} \mathrm{D}_{3}^{\circ}$ & & 14.7937 & 14.7208 & 14.7212 & & $0.690(360), 0.491(392)$ \\
\hline 361 & $4 d$ & ${ }^{1} \mathrm{P}_{1}^{0}$ & 14.5268 & 14.8073 & 14.7292 & 14.7280 & & $0.807(361),-0.587(396)$ \\
\hline 387 & $4 d$ & ${ }^{1} \mathrm{D}_{2}^{\circ}$ & & 14.9659 & 14.8706 & 14.8844 & & $0.697(358), 0.497(387), 0.403(393)$ \\
\hline 392 & $4 d$ & ${ }^{1} \mathrm{~F}_{3}^{\circ}$ & & 14.9855 & 14.8850 & 14.9027 & & $0.498(360),-0.446(392), 0.437(353)$ \\
\hline 393 & $4 d$ & ${ }^{3} \mathrm{D}_{2}^{\circ}$ & & 14.9852 & 14.8726 & 14.9081 & & $0.607(393),-0.452(387)$ \\
\hline 396 & $4 d$ & ${ }^{3} \mathrm{D}_{1}^{\circ}$ & & 15.0109 & 14.9289 & 14.9137 & & $0.782(396), 0.567(361)$ \\
\hline 524 & $4 f$ & ${ }^{3} \mathrm{D}_{1}$ & 15.5079 & 15.9458 & 15.8555 & 15.8502 & 15.3989 & $-0.997(524)$ \\
\hline 526 & $4 f$ & ${ }^{3} \mathrm{D}_{2}$ & 15.5316 & 15.9712 & 15.8804 & 15.8750 & 15.4223 & $0.962(526)$ \\
\hline 531 & $4 f$ & ${ }^{3} \mathrm{G}_{5}$ & 15.5517 & 15.9916 & 15.8994 & 15.8937 & 15.4468 & $0.997(531)$ \\
\hline 532 & $4 f$ & ${ }^{3} \mathrm{D}_{3}$ & 15.5690 & 16.0113 & 15.9192 & 15.9138 & 15.4610 & $0.881(532)$ \\
\hline 533 & $4 f$ & ${ }^{3} \mathrm{G}_{4}$ & 15.5754 & 16.0175 & 15.9224 & 15.9165 & 15.4695 & $0.837(533), 0.513(569)$ \\
\hline 549 & $4 f$ & ${ }^{3} \mathrm{G}_{3}$ & 15.6228 & 16.0699 & 15.9733 & 15.9656 & 15.5252 & $-0.737(549), 0.509(566), 0.439(568)$ \\
\hline 552 & $4 f$ & ${ }^{3} \mathrm{~F}_{4}$ & 15.6428 & 16.0934 & 15.9944 & 15.9864 & 15.7145 & $0.719(552), 0.645(569)$ \\
\hline 555 & $4 f$ & ${ }^{3} \mathrm{~F}_{2}$ & & 16.1642 & 16.0587 & 16.0516 & 15.5860 & $-0.791(555), 0.591(573)$ \\
\hline 566 & $4 \mathrm{f}$ & ${ }^{1} \mathrm{~F}_{3}$ & 15.7986 & 16.2598 & 16.1623 & 16.1571 & 15.7040 & $0.671(549), 0.524(566), 0.519(568)$ \\
\hline 568 & $4 f$ & ${ }^{3} \mathrm{~F}_{3}$ & 15.8205 & 16.2749 & 16.1744 & 16.1695 & 15.7082 & $0.635(568),-0.611(566), 0.467(532)$ \\
\hline 569 & $4 \mathrm{f}$ & ${ }^{1} \mathrm{G}_{4}$ & 15.8251 & 16.2770 & 16.1761 & 16.1701 & 15.5432 & $0.669(552),-0.560(569), 0.483(533)$ \\
\hline 573 & $4 \mathrm{f}$ & ${ }^{1} \mathrm{D}_{2}$ & 15.8515 & 16.3626 & 16.2324 & 16.2477 & 15.7635 & $0.786(573), 0.561(555)$ \\
\hline
\end{tabular}

NIST: http://physics.nist.gov/PhysRefData

GRASP: Energies from the GRASP code with 1128 level calculations.

FAC1: Energies from the FAC code with 1128 level calculations.

FAC2: Energies from the FAC code with 6202 level calculations.

MCHF: Energies of Irimia \& Froese-Fischer (2003) from the MCHF code for the levels of the 4s, and of Wagner \& House (1971) for the 4f configuration.

$a$ : Numbers of the form $M(N)$ mean that $M$ is the eigenvector of the level $N$.

experimental values. Therefore, based on this and other comparisons made among a variety of calculations, and with the available experimental compilations, we may state with confidence that our energy levels from both GRASP and FAC (with limited CI) are accurate, in magnitude, to better than $3 \%$, and those obtained from a larger CI are accurate to $\sim 1 \%$. However, the level orderings may not be conclusive as already discussed above.

\section{Radiative rates}

The absorption oscillator strength $\left(f_{i j}\right)$ and radiative rate $A_{j i}$ (in $\mathrm{s}^{-1}$ ) for a transition $i \rightarrow j$ are related by the following expression:

$f_{i j}=\frac{m c}{8 \pi^{2} e^{2}} \lambda_{j i}^{2} \frac{\omega_{j}}{\omega_{i}} A_{j i}=1.49 \times 10^{-16} \lambda_{j i}^{2}\left(\omega_{j} / \omega_{i}\right) A_{j i}$ where $m$ and $e$ are the electron mass and charge, respectively, $c$ is the velocity of light, $\lambda_{j i}$ is the transition energy/wavelength in $\AA$, and $\omega_{i}$ and $\omega_{j}$ are the statistical weights of the lower $(i)$ and upper $(j)$ levels, respectively. Similarly, the oscillator strength $f_{i j}$ (dimensionless) and the line strength $\mathrm{S}$ (in atomic unit, 1 a.u. = $6.460 \times 10^{-36} \mathrm{~cm}^{2} \mathrm{esu}^{2}$ ) are related by the following standard equations:

For the electric dipole (E1) transitions:

$A_{j i}=\frac{2.0261 \times 10^{18}}{\omega_{j} \lambda_{j i}^{3}} S^{\mathrm{E} 1} \quad$ and $\quad f_{i j}=\frac{303.75}{\lambda_{j i} \omega_{i}} S^{\mathrm{E} 1}$,

for the magnetic dipole (M1) transitions:

$A_{j i}=\frac{2.6974 \times 10^{13}}{\omega_{j} \lambda_{j i}^{3}} S^{\mathrm{M} 1} \quad$ and $\quad f_{i j}=\frac{4.044 \times 10^{-3}}{\lambda_{j i} \omega_{i}} S^{\mathrm{M} 1}$ 
for the electric quadrupole (E2) transitions:

$A_{j i}=\frac{1.1199 \times 10^{18}}{\omega_{j} \lambda_{j i}^{5}} S^{\mathrm{E} 2} \quad$ and $\quad f_{i j}=\frac{167.89}{\lambda_{j i}^{3} \omega_{i}} S^{\mathrm{E} 2}$,

and for the magnetic quadrupole (M2) transitions:

$A_{j i}=\frac{1.4910 \times 10^{13}}{\omega_{j} \lambda_{j i}^{5}} S^{\mathrm{M} 2} \quad$ and $\quad f_{i j}=\frac{2.236 \times 10^{-3}}{\lambda_{j i}^{3} \omega_{i}} S^{\mathrm{M} 2}$.

In Table 3 we present transition energies/wavelengths ( $\lambda$ in $\AA$ ), radiative rates $\left(A_{j i}\right.$ in $\left.\mathrm{s}^{-1}\right)$, oscillator strengths $\left(f_{i j}\right.$, dimensionless), and line strengths ( $S$ in a.u.), in length form only, for all 7574 electric dipole (E1) and 10815 magnetic quadrupole (M2) transitions among the lowest 250 levels of Ni XI. The indices used to represent the lower and upper levels of a transition have already been defined in Table 1. Similar results for 10754 electric quadrupole (E2) and 7499 magnetic dipole (M1) transitions are listed in Table 4. Since no other similar results are available in the literature, we hope that the presently reported $A$-values for all types of transitions will be useful in plasma modelling.

In Table 5 we compare our oscillator strengths ( $f$-values), both from GRASP and FAC, with those of Irimia \& Froese-Fischer (2003) from MCHF for the common E1 transitions. With the same CI included, as in GRASP and FAC1, the two sets of results are comparable and the discrepancies are smaller than $10 \%$, which is highly satisfactory. Similarly, the results obtained from the larger calculation (FAC2) are also comparable for the strong transitions, but differ for some weak transitions by up to $20 \%$. Furthermore, for four very weak transitions, namely $10-17\left(f \sim 10^{-4}\right), 13-17$ $\left(f \sim 10^{-3}\right), 14-143\left(f \sim 10^{-5}\right)$ and 17-132 $\left(f \sim 10^{-7}\right)$, the discrepancy is up to $50 \%$. The weak transitions are more sensitive to mixing coefficients, and hence differing amount of CI produce different $f$-values, as also discussed in detail for three Mg-like ions by Aggarwal et al. (2007). Furthermore, a larger CI generally improves the accuracy of the $f$-values for strong transitions, but is less conclusive for the weaker ones. We discuss the accuracy further below.

One of the general criterion to assess the accuracy is to compare the length and velocity forms of the $f$-or $A$-values. However, such comparisons are only desirable, but are not a fully sufficient test to assess accuracy, as different calculations (or combinations of configurations) may give comparable $f$-values in the two forms, but entirely different results in magnitude. Generally, there is a good agreement between the length and velocity forms of the $f$-values for strong transitions, but differences between the two forms can sometimes be substantial even for some very strong transitions, as discussed in detail by Aggarwal et al. (2007). Nevertheless, in Table 5 we have listed the ratio $f_{L} / f_{V}$ from our GRASP calculations. For almost all of the strong transitions $(f \geq 0.01)$ the two forms agree to within $10 \%$, but for weaker transitions (such as 14-132 and 15-143) these differ by over an order of magnitude. Overall there is no major discrepancy among our calculations from GRASP and FAC, but the earlier results of Irimia \& Froese-Fischer (2003) differ by a factor of two for some transitions, such as 1-133, 4-16, 6-17 and 7-17. Also, for the $1-143\left(3 s^{2} 3 p^{6}{ }^{1} \mathrm{~S}_{0}-3 s^{2} 3 \mathrm{p}^{5} 4 \mathrm{~s}^{1} \mathrm{P}_{1}^{\circ}\right)$ transition, their $f$-value is higher by an order of magnitude, which appears to be an anomaly. In general, we may conclude that the $f$-values of Irimia \& Froese-Fischer are comparatively less accurate, due to (perhaps) a lack of extensive CI. However, the above discussion is limited to only 52 transitions among a total of 7574 presented in Table 3. Therefore, we discuss the accuracy below for a wider range of transitions.
A comparison between our GRASP and FAC1 calculations shows that only for $28(<0.4 \%)$ strong transitions $(f \geq 0.01)$ the two sets of $f$-values differ by more than $20 \%$. Differences for these transitions are within a factor of two, except for four (51-234, 54-222, 54-234 and 76-234) for which the discrepancy is up to a factor of three. Similarly, differences between the FAC 1 and FAC $2 f$-values are more than $20 \%$ for only $154(2 \%)$ strong transitions, which indicates that the larger CI included in the FAC 2 calculations is of no appreciable advantage as far as the majority of strong transitions are concerned. Therefore, on the basis of these and earlier comparisons we may state that for a majority of strong E1 transitions, our radiative rates are accurate to better than $20 \%$. However, for the weaker transitions this assessment of accuracy does not apply.

\section{Lifetimes}

The lifetime $\tau$ for a level $j$ is defined as follows:

$\tau_{j}=\frac{1}{\sum_{i} A_{j i}}$

Since this is a measurable parameter, it provides a check on the accuracy of the calculations. Therefore, in Table 1 we have listed our calculated lifetimes, which include the contributions from four types of transitions, i.e. E1, E2, M1 and M2.

To our knowledge, no measurements of $\tau$ are available in the literature with which to compare, and the only available theoretical results are those of Irimia \& Froese-Fischer (2003) for 18 levels among the $3 s^{2} 3 p^{5} 3 d, 3 s 3 p^{6} 3 d$ and $3 s^{2} 3 p^{5} 4 s$ configurations of Ni XI. In Table 6 we compare the lifetimes for the common levels for which the discrepancy is up to three orders of magnitude. There are three main reasons for these large differences. Firstly, the two sets of $A$ - (or $f$-) values are different as already seen from Table 5 . This accounts for differences up to $\sim 50 \%$ for some of the levels, such as 3 and $4\left(3 s^{2} 3 p^{5} 3 d^{3} P_{1,2}^{\circ}\right)$. Secondly, Irimia \& Froese-Fischer have not calculated $A$-values for some types of transitions, which happen to have the dominant contribution. For example, for level $6\left(3 s^{2} 3 p^{5} 3 d^{3} F_{3}^{\circ}\right)$ their determination of $\tau$ is based solely on the 1-6 E3 transition for which $A=0.05443 \mathrm{~s}^{-1}$, whereas we find that the dominant contribution comes from the 5-6 M1 transition for which $A=2.08 \mathrm{~s}^{-1}$, as shown in Tables 3 and 4. Finally, and in particular for the higher excited levels of the $3 s^{2} 3 p^{5} 4 s$ configuration, they have not calculated $A$-values from all the lower levels, which are required for the determination of $\tau$, as shown in Eq. (6). This is the main reason for the difference of three orders of magnitude in the lifetimes of the levels of the $3 s^{2} 3 p^{5} 4$ s configuration. To conclude, we may state that the lifetimes of the levels listed in Table 1 are the most accurate currently available. However, further calculations or future measurements, particularly for the $\left(3 s^{2} 3 p^{5} 3 d\right)$ ${ }^{3} \mathrm{~F}_{4,3,2}^{\circ}$ levels for which the $\tau$ values are $67.76,0.434$ and $0.065 \mathrm{~s}$, respectively, will be helpful in further assessing the accuracy of our results.

\section{Excitation rates}

Excitation rates, along with energy levels and radiative rates, are required for plasma modelling, and are determined from the collision strengths $(\Omega)$. Since the threshold energy region is dominated by numerous closed-channel (Feshbach) resonances, as demonstrated in Figs. 5 and 6 of Aggarwal \& Keenan (2007), values of $\Omega$ need to be calculated in a fine energy mesh in order to accurately account for their contribution. Furthermore, in 
Table 5. Comparison of oscillator strengths ( $f$-values) for some transitions of Ni XI. $a \pm b \equiv a \times 10^{ \pm b}$.

\begin{tabular}{|c|c|c|c|c|c|c|c|}
\hline$I$ & $J$ & Transition & GRASP & $f_{L} / f_{V}$ & FAC1 & FAC2 & MCHF \\
\hline 1 & 3 & & $4.447-4$ & $9.9-1$ & $4.568-4$ & $4.515-4$ & $3.486-4$ \\
\hline 1 & 10 & & $8.591-3$ & 9.9-1 & $8.882-3$ & $8.744-3$ & $7.538-3$ \\
\hline 1 & 13 & & $2.660-0$ & $1.0+0$ & $2.617-0$ & $2.632-0$ & $2.528-0$ \\
\hline 1 & 133 & & $1.295-1$ & $1.0+0$ & $1.175-1$ & $1.110-1$ & $6.985-1$ \\
\hline 1 & 143 & & $1.856-1$ & $1.0+0$ & $1.651-1$ & $1.563-1$ & $1.968-0$ \\
\hline 2 & 14 & & $6.198-2$ & $1.1+0$ & $6.367-2$ & $6.015-2$ & $1.038-1$ \\
\hline 3 & 14 & & $2.036-2$ & $1.1+0$ & $2.100-2$ & $1.965-2$ & $3.245-2$ \\
\hline 3 & 15 & & $4.232-2$ & $1.1+0$ & $4.338-2$ & $4.138-2$ & $6.931-2$ \\
\hline 3 & 17 & & $2.754-4$ & $1.0+0$ & $2.946-4$ & $3.258-4$ & $3.666-4$ \\
\hline 4 & 14 & & $1.540-3$ & $1.0+0$ & $1.592-3$ & $1.455-3$ & $2.263-3$ \\
\hline 4 & 15 & & $1.465-2$ & $1.1+0$ & $1.511-2$ & $1.411-2$ & $2.178-2$ \\
\hline 4 & 16 & & 4.779-2 & $1.1+0$ & 4.898-2 & $4.698-2$ & 7.347-2 \\
\hline 4 & 17 & & $5.479-4$ & $6.9-1$ & $5.819-4$ & $5.232-4$ & $1.180-3$ \\
\hline 5 & 16 & & $3.851-2$ & $5.7-1$ & $3.963-2$ & $3.333-2$ & $4.773-2$ \\
\hline 6 & 15 & & $3.587-2$ & $5.8-1$ & $3.684-2$ & $3.122-2$ & $4.406-2$ \\
\hline 6 & 16 & & $9.423-4$ & $2.7-1$ & $9.528-4$ & 7.657-4 & $1.160-3$ \\
\hline 6 & 17 & & $6.067-4$ & 9.4-1 & $6.563-4$ & $4.738-4$ & $1.218-3$ \\
\hline 7 & 14 & & $3.371-2$ & $5.8-1$ & $3.460-2$ & $2.929-2$ & $4.045-2$ \\
\hline 7 & 15 & & $1.255-3$ & $2.8-1$ & $1.250-3$ & $9.865-4$ & $1.380-3$ \\
\hline 7 & 16 & & $1.013-4$ & $1.7+0$ & $1.103-4$ & $1.075-4$ & $1.465-4$ \\
\hline 7 & 17 & & $1.809-3$ & 4.9-1 & $1.927-3$ & $1.604-3$ & $3.816-3$ \\
\hline 8 & 15 & & $1.983-3$ & 9.8-1 & $2.051-3$ & $1.686-3$ & $2.077-3$ \\
\hline 8 & 16 & & $2.165-2$ & $9.3-1$ & $2.199-2$ & $1.916-2$ & $2.388-2$ \\
\hline 8 & 17 & & $9.541-3$ & $7.7-1$ & $1.015-2$ & $8.402-3$ & $1.392-2$ \\
\hline 9 & 14 & & $3.050-4$ & $1.3-2$ & $3.297-4$ & $3.273-4$ & $7.822-4$ \\
\hline 9 & 15 & & $1.403-2$ & $8.0-1$ & $1.449-2$ & $1.257-2$ & $1.473-2$ \\
\hline 9 & 16 & & $2.207-3$ & $8.9-1$ & $2.274-3$ & $1.909-3$ & $1.726-3$ \\
\hline 9 & 17 & & $1.365-2$ & $4.7-1$ & $1.404-2$ & $1.132-2$ & $2.555-2$ \\
\hline 10 & 14 & & $2.273-2$ & 9.1-1 & $2.333-2$ & $2.004-2$ & $2.366-2$ \\
\hline 10 & 15 & & $1.285-2$ & $9.7-1$ & $1.321-2$ & $1.169-2$ & $1.557-2$ \\
\hline 10 & 17 & & $1.040-4$ & $5.5-1$ & $1.176-4$ & $7.531-5$ & $2.430-4$ \\
\hline 11 & 14 & & $2.184-3$ & $9.7-1$ & $2.235-3$ & $1.901-3$ & $2.119-3$ \\
\hline 11 & 15 & & $1.094-2$ & $9.1-1$ & $1.116-2$ & $9.482-3$ & $1.201-2$ \\
\hline 11 & 16 & & $9.692-3$ & $1.0+0$ & $9.989-3$ & $9.107-3$ & $1.364-2$ \\
\hline 11 & 17 & & $1.014-2$ & $5.1-1$ & $1.050-2$ & $8.633-3$ & $1.406-2$ \\
\hline 12 & 15 & & $5.900-5$ & $3.6-1$ & $5.807-5$ & $4.358-5$ & $1.125-4$ \\
\hline 12 & 16 & & $1.370-2$ & $8.5-1$ & $1.435-2$ & $1.228-2$ & $1.474-2$ \\
\hline 12 & 17 & & $1.595-2$ & 7.3-1 & $1.632-2$ & $1.390-2$ & $2.290-2$ \\
\hline 13 & 14 & & $2.584-5$ & $7.5-1$ & & & $2.618-5$ \\
\hline 13 & 15 & & $1.943-4$ & $5.8-1$ & $2.077-4$ & $1.898-4$ & $1.783-4$ \\
\hline 13 & 17 & & $8.130-3$ & $2.3-1$ & $8.555-3$ & $5.254-3$ & $8.143-3$ \\
\hline 14 & 132 & & $8.747-6$ & $5.7-2$ & $8.685-6$ & $8.774-6$ & $4.150-5$ \\
\hline 14 & 133 & & $8.460-5$ & $5.5-2$ & $8.172-5$ & $8.586-5$ & $4.385-4$ \\
\hline 14 & 140 & & $2.183-4$ & $6.5-2$ & $2.036-4$ & $2.498-4$ & $7.898-4$ \\
\hline 14 & 143 & & $8.125-5$ & $8.7-2$ & $6.770-5$ & $9.602-5$ & $1.637-4$ \\
\hline 15 & 132 & & 7.893-5 & $4.8-2$ & $7.842-5$ & $8.014-5$ & $3.936-4$ \\
\hline 15 & 133 & & $1.384-4$ & $6.2-2$ & $1.333-4$ & $1.399-4$ & $6.990-4$ \\
\hline 15 & 143 & & $1.421-4$ & $4.7-2$ & $1.333-4$ & $1.874-4$ & $4.720-4$ \\
\hline 16 & 132 & & $3.308-4$ & $4.2-2$ & $3.270-4$ & $3.381-4$ & $1.723-3$ \\
\hline 17 & 132 & & $3.048-7$ & $4.2-1$ & $2.678-7$ & $3.527-7$ & $2.107-6$ \\
\hline 17 & 133 & & $8.640-5$ & $8.2-5$ & $8.756-5$ & 8.664-5 & $1.111-3$ \\
\hline 17 & 143 & & $9.128-5$ & $8.8-4$ & $9.560-5$ & $7.613-5$ & $2.660-3$ \\
\hline
\end{tabular}

GRASP: Calculations from the GRASP code with 1128 levels.

FAC1: Calculations from the FAC code with 1128 levels.

FAC2: Calculations from the FAC code with 6202 levels.

MCHF: Calculations of Irimia \& Froese-Fischer (2003) from the MCHF code.

a hot plasma electrons have a wide distribution of velocities, and therefore values of $\Omega$ are generally averaged over a Maxwellian distribution as follows:

$\Upsilon\left(T_{\mathrm{e}}\right)=\int_{0}^{\infty} \Omega(E) \exp \left(-E_{j} / k T_{\mathrm{e}}\right) \mathrm{d}\left(E_{j} / k T_{\mathrm{e}}\right)$,

where $k$ is Boltzmann constant, $T_{\mathrm{e}}$ is electron temperature in $\mathrm{K}$, and $E_{j}$ is the electron energy with respect to the final (excited) state. Once the value of $\Upsilon$ is known the corresponding results for the excitation $q(i, j)$ and de-excitation $q(j, i)$ rates can be easily obtained from the following equations:

$q(i, j)=\frac{8.63 \times 10^{-6}}{\omega_{i} T_{\mathrm{e}}^{1 / 2}} \Upsilon \exp \left(-E_{i j} / k T_{\mathrm{e}}\right) \mathrm{cm}^{3} \mathrm{~s}^{-1}$ 
Table 6. Comparison of lifetimes (in s) for some levels of Ni XI. $a \pm b \equiv a \times 10^{ \pm b}$.

\begin{tabular}{rllcc}
\hline \hline Index & Configuration & Level & Present results & Irimia \& Froese-Fischer (2003) \\
\hline 3 & $3 \mathrm{~s}^{2} 3 \mathrm{p}^{5} 3 \mathrm{~d}$ & ${ }^{3} \mathrm{P}_{1}^{\circ}$ & $4.458-08$ & $6.724-08$ \\
4 & $3 \mathrm{~s}^{2} 3 \mathrm{p}^{5} 3 \mathrm{~d}$ & ${ }^{3} \mathrm{P}_{2}^{\circ}$ & $6.421-03$ & $1.021-02$ \\
6 & $3 \mathrm{~s}^{2} 3 \mathrm{p}^{5} 3 \mathrm{~d}$ & ${ }^{3} \mathrm{~F}_{3}^{\circ}$ & $4.338-01$ & $1.837+01$ \\
7 & $3 \mathrm{~s}^{2} 3 \mathrm{p}^{5} 3 \mathrm{~d}$ & ${ }^{3} \mathrm{~F}_{2}^{\circ}$ & $6.483-02$ & $2.385-01$ \\
8 & $3 \mathrm{~s}^{2} 3 \mathrm{p}^{5} 3 \mathrm{~d}$ & ${ }^{3} \mathrm{D}_{3}^{\circ}$ & $1.445-02$ & $1.825-00$ \\
9 & $3 \mathrm{~s}^{2} 3 \mathrm{p}^{5} 3 \mathrm{~d}$ & ${ }^{1} \mathrm{D}_{2}^{\circ}$ & $4.314-03$ & $2.777-02$ \\
10 & $3 \mathrm{~s}^{2} 3 \mathrm{p}^{5} 3 \mathrm{~d}$ & ${ }^{3} \mathrm{D}_{1}^{\circ}$ & $1.777-09$ & $2.377-09$ \\
11 & $3 \mathrm{~s}^{2} 3 \mathrm{p}^{5} 3 \mathrm{~d}$ & ${ }^{3} \mathrm{D}_{2}^{\circ}$ & $3.479-03$ & $3.234-01$ \\
12 & $3 \mathrm{~s}^{2} 3 \mathrm{p}^{5} 3 \mathrm{~d}$ & ${ }^{1} \mathrm{~F}_{3}^{\circ}$ & $2.454-03$ & $8.150-01$ \\
13 & $3 \mathrm{~s}^{2} 3 \mathrm{p}^{5} 3 \mathrm{~d}$ & ${ }^{1} \mathrm{P}_{1}^{\circ}$ & $3.557-12$ & $4.264-12$ \\
14 & $3 \mathrm{~s} 3 \mathrm{p}^{6} 3 \mathrm{~d}$ & ${ }^{3} \mathrm{D}_{1}$ & $9.665-11$ & $6.701-11$ \\
15 & $3 \mathrm{~s} 3 \mathrm{p}^{6} 3 \mathrm{~d}$ & ${ }^{3} \mathrm{D}_{2}$ & $9.649-11$ & $6.791-11$ \\
16 & $3 \mathrm{~s} 3 \mathrm{p}^{6} 3 \mathrm{~d}$ & ${ }^{3} \mathrm{D}_{3}$ & $9.587-11$ & $6.903-11$ \\
17 & $3 \mathrm{~s} 3 \mathrm{p}^{6} 3 \mathrm{~d}$ & ${ }^{1} \mathrm{D}_{2}$ & $1.967-10$ & $3.163-10$ \\
132 & $3 \mathrm{~s}^{2} 3 \mathrm{p}^{5} 4 \mathrm{~s}$ & ${ }^{3} \mathrm{P}_{2}^{\circ}$ & $7.993-09$ & $3.638-12$ \\
133 & $3 \mathrm{~s}^{2} 3 \mathrm{p}^{5} 4 \mathrm{~s}$ & ${ }^{3} \mathrm{P}_{1}^{\circ}$ & $1.990-11$ & $4.827-14$ \\
140 & $3 \mathrm{~s}^{2} 3 \mathrm{p}^{5} 4 \mathrm{~s}$ & ${ }^{3} \mathrm{P}_{0}^{\circ}$ & $6.167-09$ & $1.364-14$ \\
143 & $3 \mathrm{~s}^{2} 3 \mathrm{p}^{5} 4 \mathrm{~s}$ & ${ }^{1} \mathrm{P}_{1}^{\circ}$ & $1.342-11$ & \\
\hline
\end{tabular}

and

$q(j, i)=\frac{8.63 \times 10^{-6}}{\omega_{j} T_{\mathrm{e}}^{1 / 2}} \Upsilon \mathrm{cm}^{3} \mathrm{~s}^{-1}$,

where $\omega_{i}$ and $\omega_{j}$ are the statistical weights of the initial $(i)$ and final $(j)$ states, respectively, and $\mathrm{E}_{i j}$ is the transition energy. The contribution of resonances may enhance the values of $\Upsilon$ over those of the background values of collision strengths $\left(\Omega_{B}\right)$, especially for the forbidden transitions, by up to a factor of ten (or even more) depending on the transition and/or the temperature. Similarly, values of $\Omega$ need to be calculated over a wide energy range (above thresholds) in order to obtain convergence of integral in Eq. (7), as demonstrated in Fig. 7 of Aggarwal \& Keenan.

In our earlier paper (Aggarwal \& Keenan 2007), we reported results for both $\Omega$ and $\Upsilon$, but only for the resonance transitions among the 17 levels of the $3 s^{2} 3 p^{6}, 3 s^{2} 3 p^{5} 3 d$ and $3 s^{3} p^{6} 3 d$ configurations. In Table 7 we report results of $\Upsilon$ for all transitions among these levels. The details of our calculations have already been described in that paper, but we recapitulate the main points here. For the computations of $\Omega$, we employed the fully relativistic Dirac Atomic R-matrix Code (DARC) of Norrington \& Grant (2007), as implemented by Ait-Tahar et al. (1996). The $R$-matrix radius was adopted to be $2.97 \mathrm{au}$, and 19 continuum orbitals were included for each channel angular momentum for the expansion of the wavefunction. This allowed us to compute values of $\Omega$ up to an energy of $220 \mathrm{Ryd}$, more than sufficient to determine the values of $\Upsilon$ up to a temperature of $10^{7} \mathrm{~K}$. Furthermore, in order to obtain convergence of $\Omega$ for all transitions and at all energies, we included all partial waves with angular momentum $J \leq 39.5$. To account for the inclusion of higher neglected partial waves, we also included a top-up, based on Coulomb-Bethe approximation for allowed transitions and geometric series for others. Similarly, to account for the contribution of resonances we calculated values of $\Omega$ in a fine energy mesh $(\Delta E \leq 0.002$ Ryd) throughout the thresholds region.

To our knowledge, no other similar results, either for $\Omega$ or $\Upsilon$, are available in the literature with which to compare. However, based on a variety of comparisons, including a parallel calculation performed with the Flexible Atomic Code (FAC) of $\mathrm{Gu}$ (2003), our results for $\Omega$, and subsequently for $\Upsilon$, were assessed to be accurate to better than $25 \%$ - see Aggarwal \& Keenan (2007) for details. This accuracy can be further improved by adopting a larger ion model, as already demonstrated for the energy levels and radiative rates in Sects. 2 and 3, respectively. A larger model, say of about 130 levels, will not only improve the accuracy of the $\Upsilon$ values by accounting for the resonances arising from the higher excited levels, but will also be useful for modelling of plasmas (Liedahl 2000). However, such a collisional calculation will require a considerable amount of computational effort, but should be possible to perform in the future. Until then, the values of $\Upsilon$ reported in Table 7 can be employed with confidence in the analysis of Ni XI transitions observed from high temperature plasmas (Mattioli et al. 2004).

\section{Conclusions}

In this paper we have presented results for energy levels and radiative rates for four types of transitions (E1, E2, M1 and M2) among the lowest 250 levels of Ni XI. These 250 levels include all those of the $3 s^{2} 3 p^{6}, 3 s^{2} 3 p^{5} 3 d, 3 s 3 p^{6} 3 d, 3 p^{4} 3 d^{2}, 3 s^{2} 3 p^{5} 4 s$ and $3 s^{2} 3 p^{5} 4 p$ configurations. Additionally, lifetimes of all the levels have been reported, although no measurements are available with which to compare. However, the corresponding results for excitation rates are presented only for transitions among the lowest 17 levels of the $3 s^{2} 3 p^{6}, 3 s^{2} 3 p^{5} 3 d$ and $3 s 3 p^{6} 3 d$ configurations. Therefore, a larger collisional calculation involving a considerably greater number of levels will be desirable in future.

Based on a variety of comparisons, our energy levels are assessed to be accurate to better than $3 \%$, and the results for radiative rates, oscillator strengths, line strengths, and lifetimes are assessed to be accurate to better than $20 \%$ for a majority of strong transitions (levels). The accuracy for excitation rates is comparatively poor $(\sim 25 \%)$, which can only be improved by considering a larger model.

Acknowledgements. This work has been financed by the Engineering and Physical Sciences and Science and Technology Facilities Councils of the United Kingdom, and F.P.K. is grateful to AWE Aldermaston for the award of a William Penney Fellowship. 


\section{References}

Aggarwal, K. M., \& Keenan, F. P. 2006, A\&A, 450, 1249

Aggarwal, K. M., \& Keenan, F. P. 2007, Eur. Phys. J. D, in press

Aggarwal, K. M., Keenan, F. P., \& Msezane, A. Z. 2003, ADNDT, 85, 453

Aggarwal, K. M., Tayal, V., Gupta, G. P., \& Keenan, F. P. 2007, ADNDT, 93, 615

Ait-Tahar, S., Grant, I. P., \& Norrington, P. H. 1996, Phys. Rev. A, 54, 3984 Berrington, K. A., Eissner, W. B., \& Norrington, P. H. 1995, Comput. Phys. Commun., 92, 290

Dyall, K. G., Grant, I. P., Johnson, C. T., Parpia, F. A., \& Plummer, E. P. 1989, Comput. Phys. Commun., 55, 424

Edlen, B., \& Smitt, R. 1978, Sol. Phys., 57, 329

Fawcett, B. C., Cowan, R. D., \& Hayes, R. W. 1972, J. Phys. B, 5, 2143

Feldman, U., Fraenkel, B. S., \& Hoory, S. 1965, ApJ, 142, 719

Grant, I. P., McKenzie, B. J., Norrington, P. H., Mayers, D. F., \& Pyper, N. C. 1980, Comput. Phys. Commun., 21, 207
Gu, M. F. 2003, ApJ, 582, 1241

Hibbert, A. 1975, Comput. Phys. Commun., 9, 141

Irimia, A., \& Froese-Fischer, C. 2003, available at http://www.vuse.vanderbilt.edu/ cff/mchf_collection/ Liedahl, D. A. 2000, Atomic Data Needs for X-ray Astronomy available at http://heasarc.gsfc.nasa.gov/docs/heasarc/atomic/

Magnant-Crifo, F. 1975, Sol. Phys., 41, 109

Mattioli, M., Fournier, K. B., Coffey, I., \& Contributors to the EFDA-JET Work Programme 2004, J. Phys. B, 37, 13

Norrington, P. H., \& Grant, I. P. 2007, Comput. Phys. Commun., in preparation Raassen, A. J. J., Mewe, R., Audard, M., et al. 2002, A\&A, 389, 228

Raassen, A. J. J., Ness, J. U., Mewe, R., et al. 2003, A\&A, 400, 671

Sandlin, G. D., \& Tousey, R. 1979, ApJ, 227, L107

Svensson, L. A., Ekberg, J. O., \& Edlen, B. 1974, Sol. Phys., 34, 173

Verma, N., Jha, A. K. S., \& Mohan, M. 2007, Eur. Phys. J. D, 42, 235

Wagner, W. J., \& House, L. L. 1971, ApJ, 166, 683 